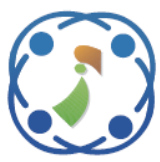

\title{
Driving Preference Metric-Aware Control for Self-Driving Vehicles
}

\author{
Il Bae ${ }^{1} \quad$ Jaeyoung Moon ${ }^{1} \quad$ Shiho Kim ${ }^{1 *}$ \\ ${ }^{1}$ Seamless Transportation Lab, School of Integrated Technology, College of Engineering, \\ Yonsei University, Seoul 03722, South Korea. \\ * Corresponding author's Email: shiho@yonsei.ac.kr
}

\begin{abstract}
One of crucial concern of autonomous vehicles is that driver may experience discomfort, if the driving pattern of the vehicle is quite different from her/his personal driving style. This study focuses on an autonomous vehicle control based on user's driving preference adopted to provide a personalized and a familiar driving experience to autonomous vehicle users. For this purpose, we defined a driver's driving preference metric (DPM) to reflect their own driving style by specifying a preferred lateral and longitudinal acceleration region. Moreover, we also proposed a time optimal speed planning that utilize the DPM control parameters extracted from a GG diagram to provide a DPM-aware maneuvering for a personalized autonomous driving. The performance of the proposed planning strategy and a combined vehicle controller were verified using on-road experimental tests. The experimental result shows that the proposed DPM-aware system can control the lateral and longitudinal acceleration of autonomous vehicle within $28.7 \%$ of peak error tolerance from the specified criterion of DPM.
\end{abstract}

Keywords: Autonomous vehicles, Driver preference metric, Personalized autonomous driving, Automated driving style, Driving comfort, Vehicular controller.

\section{Introduction}

Autonomous vehicles are convenient since they subduct the driving workload, whereby driving a vehicle can be accomplished without paying attention to the roadway environment. In addition to the convenience, the key feature of self-driving is that it can prevent accidents which are mainly caused by drivers' mistakes. For autonomous vehicles of level-3 or higher, a driver sitting in the cabin does not need to engage in driving maneuvers, so, the driver becomes a passenger similar to one of users in public transportation vehicles [1]. Autonomous driving system allows the driver to perform other productive tasks, or engage in relaxation or various entertainment activities [2], such as games, videos, the internet, and etc. However, one crucial concern for drivers in autonomous vehicles is that they may experience discomfort, or they may be prone to an increased likelihood of motion sickness, if the driving pattern of the vehicle is quite different from his/her own driving styles, or if it deviates from the favorable driving patterns.

Despite notable achievements have been published in the past few decades regarding the development of autonomous vehicle controllers [36], yet there is a lack of investigations on the control of the self-driving vehicle in accordance to the individual driver's favorable driving style. Reflecting individual driving styles or personalized preferences has become an important issue in the implementation for self-driving cars in terms of the human factors [7-8].

A number of articles have been published on modelling driver behaviors and classification of the driving style. The driving style is an intricate concept associated with components of the driving context and nonlinear, uncertainty, or the random characteristics of the behaviors of human drivers. However, according to [9-15], the recorded longitudinal and lateral acceleration values have 
been revealed as the key parameters that specify the preferred driving style as a response to dynamic changes to the driving conditions. The longitudinal and lateral acceleration values are the combined results of vehicular dynamics and driver maneuvers according to his/her perception and acceptance level of risks, in response to dynamic consecutive changes of the driving conditions and situations. Therefore, defining a driver's driving style based on accelerations is a well-known and a reasonable approach.

Furthermore, the driver's driving style can be represented graphically on the GG diagram, which is the two-dimensional diagram of lateral and longitudinal accelerations [9, 10]. The GG diagram is used to define the performance envelope of a car $[11,16]$ and it can also be used to refine the abilities of a driver, or to characterize the user driving styles. Typically, the driver's driving style have been classified into three categories that include aggressive (or sporty), mild (or moderate), and calm (or cautious) types [14-16]. We have been motivated that an autonomous driving system should provide control flexibilities and automated driving options for a familiar and a comfortable automated driving experience of each user.

The first contribution of this study is that we propose a system architecture to reflect driving preference based on the preferred longitudinal and lateral acceleration region to provide a personalized driving experience in autonomous vehicles. Seconds, this study provides an autonomous vehicle system which can interact with drivers based on the GG diagram interface for the customization. Finally, this research presents a real experimental result of the implemented controller, which can control selfdriving vehicles in accordance to the specified region of admissible accelerations.

This paper has been organized into 6 sections. Section 2 explains driver's preference metric based on accelerations and jerks of drivers using GG diagram. Section 3 presents an optimal speed planning method with the driver's preference-aware control system to provide a personalized driving. Experimental results and discussions are presented in Sections 4 and 5. The conclusions are finally drawn in Section 6.

\section{Representation of driving preference metric (DPM) on GG diagram}

Typically, the GG diagram is used to represent the performance envelop of a car with a twodimensional graph that depicts the longitudinal and lateral accelerations along the vertical and horizontal axes. The maximum friction force denotes the capability of the exerted vehicular grip on the surface of the road to ensure stable movement. Based on the assumption that the grip is fairly isotropic in all directions, the maximum frictional force boundary can be approximated by a circle. This circle is the maximum control boundary of the vehicle, and is used to ensure the stability of movement without skidding. The maximum acceleration capability, braking capability, and maximum tire grip, determine the performance envelop of the vehicle.

Fig. 1 shows an example of a friction circle and the performance envelop of a vehicle. The maximum acceleration capability is a property that is almost the same as Zero-to-100 performance limit of the vehicle, and is usually smaller than the friction limit of the tire grip so that the vehicle performance envelop yields an asymmetrical shape with respect to the axis of the longitudinal acceleration. The variation of the driver's lateral and longitudinal acceleration values displayed on the GG diagram show an objective index type in regard to the "driver's capability envelope," or in regard to the graphical representation of the user's driving style [9].

For instance, racing drivers manipulate the vehicular dynamics almost up to the end-range of the performance envelop, whereas normal drivers cannot utilize the full available capability of the vehicular performance. Normal drivers may fear for vehicular instability, and it is thus difficult to brake and turn at the same time. Typical criteria for longitudinal and lateral acceleration parameters for normal, aggressive, and extremely aggressive drivers, are plotted on the GG diagram, as shown in Fig. 1. The asymmetrical elliptically shaped curve enclosing the parameters set by the referred criterion represents the driver's performance envelop, which denotes the approximate limit of the driver's maximum capability in maneuvering the vehicle during dynamic motion. Statistics on driving data revealed that each driver possesses a unique lateral and longitudinal acceleration profile with the maximum values being considerably below the adherence limit of grip of tire on the road [10]. Moreover, approximately $40 \%$ of the acceleration motion control occurs in conditions of uniform speed (i.e., at zero acceleration), and approximately $90 \%$ of longitudinal accelerations occur within the range of $0-1.5 \mathrm{~m} / \mathrm{s}^{2}$ for normal drivers $[10,17]$. 


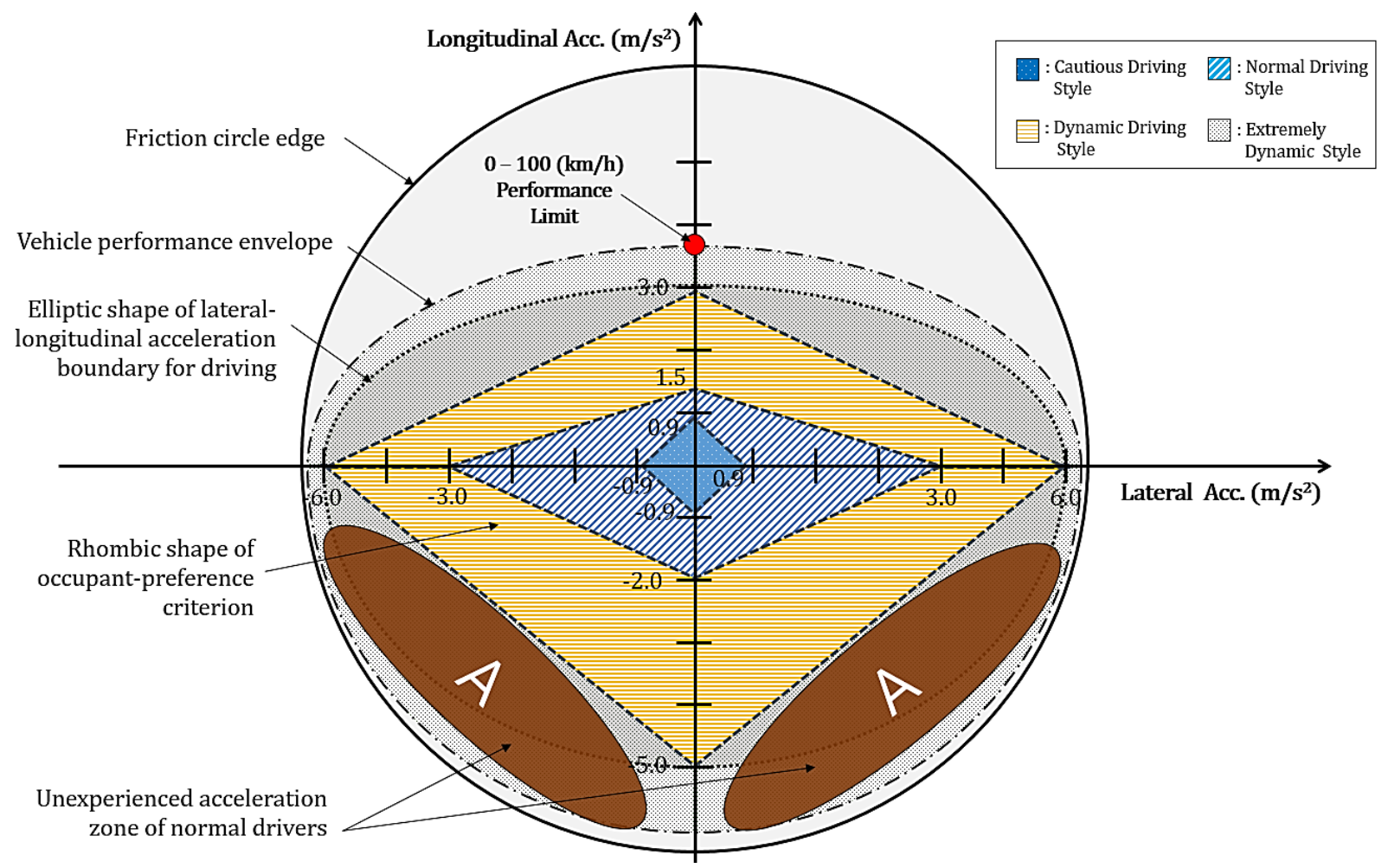

Figure. 1 Proposed driver's preference metric (DPM) display on the GG diagram along with friction circle and performance envelop of the vehicle. Note that the units of the $\mathrm{x}$ - and $\mathrm{y}$-axes are in $\mathrm{m} / \mathrm{s}^{2}$, instead of acceleration of gravity units

Recorded longitudinal and lateral acceleration values are the means of the combined results of driver's preferences and perceived risk level corresponding to the dynamic motion at a given environment. Measurement data from three drivers [10] with different driving skills and experience revealed that the maximum values of the lateral acceleration depend on the driving skill and experience as follows,

1) Normal driver : $\pm 3 \mathrm{~m} / \mathrm{s}^{2}$,

2) Aggressive driver : $\pm 6 \mathrm{~m} / \mathrm{s}^{2}$,

3) Driver with racing experience $: \pm 8 \mathrm{~m} / \mathrm{s}^{2}$.

This study proposes to define the driver's preference metric with a set of selection parameters, including the forward longitudinal acceleration/ deceleration, lateral acceleration of the left and right sides, longitudinal- and lateral-jerk (derivative of acceleration). Then, the proposed DPM is a set of criteria represented by

$$
D P M=\left\{a_{+x_{D P M}}, a_{-x_{D P M}},\left|a_{y_{D P M}}\right|,\left|z_{x_{D P M}}\right|,\left|z_{y_{D P M}}\right|\right\}
$$

Herein, each of the elements in the listed DPM expression respectively denote the vehicular longitudinal acceleration $\left(a_{+x_{D P M}}\right)$, longitudinal deceleration $\left(a_{-x_{D P M}}\right)$, absolute value of lateral acceleration $\left(\left|a_{y_{D P M}}\right|\right)$, and the absolute value of the longitudinal and lateral jerks $\left(\left|z_{x_{D P M}}\right|,\left|z_{y_{D P M}}\right|\right)$. It can be noted that the axes units are in $\mathrm{m} / \mathrm{s}^{2}$ instead of using a normalized acceleration scale using gravity units, because the DPM criterion is represented in units of $\mathrm{m} / \mathrm{s}^{2}$.

Statistics on driving style revealed that normal drivers perform a smooth transition from deceleration rather than simultaneous abrupt cornering and deceleration, thereby resulting in a self-limited small amount of lateral acceleration during braking. The two areas, labelled using the letter " $A$ ", indicate the acceleration zones rarely observed for ordinary non-racing drivers. In Fig. 1, the driver's preference criterion is modelled as an asymmetric rhombic shape by connecting each interception point with the $\mathrm{x}$ - and $\mathrm{y}$-axes with straight lines. Herein, we model the DPM as the favorable acceleration zone for a driver of the selfdriving car. 


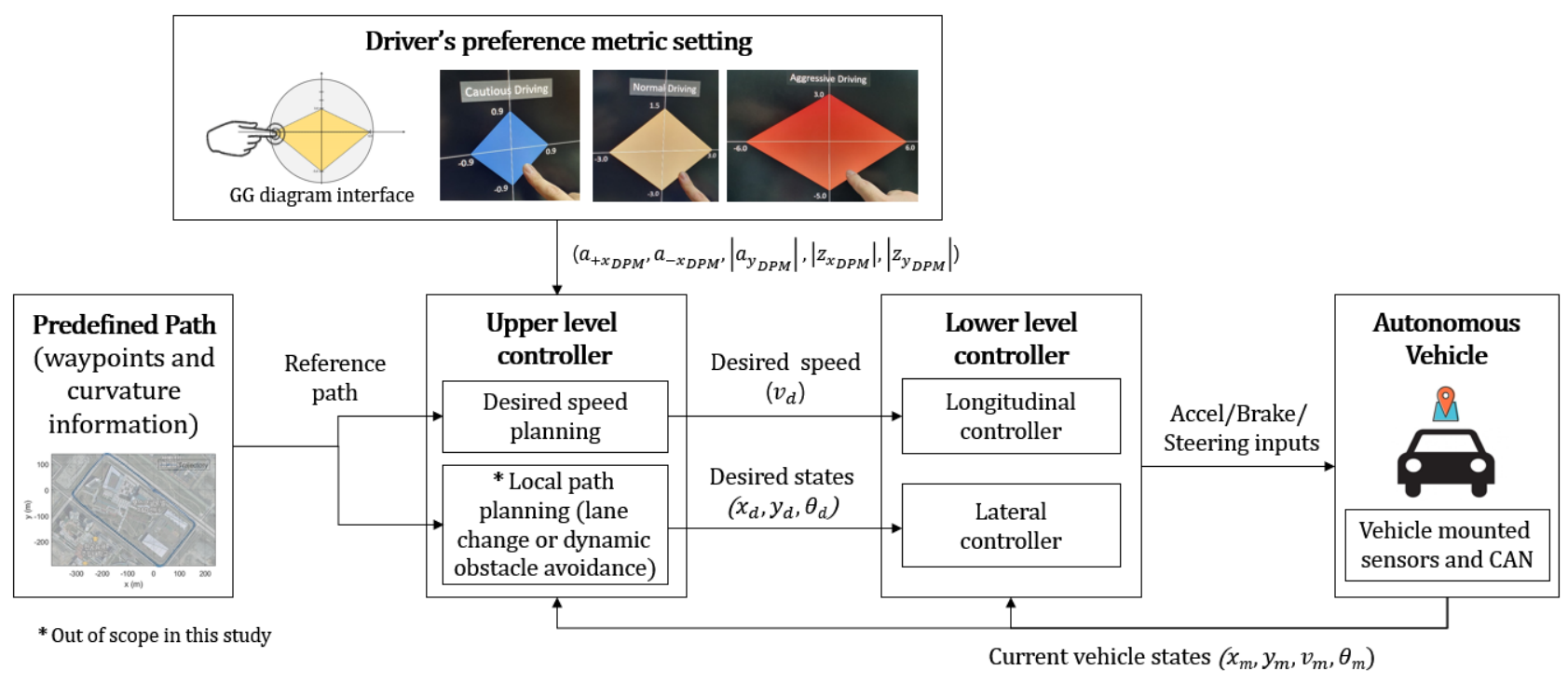

Figure. 2 Block diagram of the proposed driver's preference metric control system

Fig. 1 also depicts the region of proposed DPM for a cautious [18], normal, dynamic, and extremely dynamic style in this study. If we assume that a driver of the fully autonomous self-driving vehicle can select his/her driving preference, the preferred driving style can be interpreted as a preference metric that the driver might want for experience during riding a car. In addition, the magnitude of jerks was considered $0.6 \mathrm{~m} / \mathrm{s}^{3}$ by the comfort criteria of previous studies $[1,18]$.

\section{Driver's driving preference based control system for autonomous vehicles}

\subsection{Driver's preference metric-aware system}

Fig. 2 presents the block diagram of the proposed control system for a driver's driving preference-aware autonomous vehicle. The main components of the proposed control system are the blocks for DPM selection for velocity planning, and for integrated longitudinal lateral control input to the autonomous vehicle. The user can select his/her own criteria of DPM parameters to meet his/her driving preferences. The criteria and parameters of the typical driver include cautious, normal, dynamic, and extremely dynamic styles, and are stored in the DB of the DPM as recommended reference values available for selection. It is also possible to customize with the user's preference parameters, rather than select among the recommended reference values in a typical pattern.

The selected DPM parameters are used as a criterion for determining the maximum allowable ranges of accelerations and jerks during velocity planning on a predefined reference path. In regard to the desired reference path, the forward velocity can be planned so that the lateral acceleration does not exceed the bounded range of the selected DPM by negotiating velocity with curvature for the specific road under consideration.

If a lane change or obstacle avoidance is required while driving along a predetermined route and in accordance to the map, the local path may need to be generated in real time. In this case, by considering the current driving speed of the vehicle, a path is dynamically generated in the trajectory planning block in real time that satisfies the range of the lateral acceleration of the DPM. However, the detail of the local dynamic path planning is beyond research scope of this study.

The reference path and state variables of the vehicle determined in the upper level controller block become the input to the lower level controller. The combined longitudinal and lateral controller module calculates the required steering control, throttle, and brake inputs of the vehicle to track the reference path with the desired vehicular input state. The state variables of the vehicle, such as its current position, velocity, and yaw and steering angles, are measured by the mounted vehicular sensors and CAN at every predefined period (i.e., every $0.1 \mathrm{~s}$ ), and are transferred as the input variables to the upper level controller block and to the lower level controller block, simultaneously.

\subsection{Time optimal desired speed planning based on driver's driving preference}

In the case of a straight road, the desired speed can be determined by taking into account the speed 
limit and the safe braking distance to prevent collisions with obstacles at the front of the car. However, on curved roads, the lateral acceleration is proportional to the centrifugal force of the vehicle, which in turn, is proportional to the square of the speed and to the curvature of the road. In order to meet the criterion of lateral acceleration and jerk, a vehicle traveling at high speeds must decelerate before it enters a curved road, while concurrently satisfying the criterion of longitudinal acceleration and jerk. This study exploited a simultaneous approach, based on the interior-point-method (IPM) to optimize the desired speed that minimized the travel time of the route, while vehicle states simultaneously complied with the criterion of DPM in a given route. The proposed simultaneous trajectory and speed co-planning method was used to calculate the optimum driving speed that satisfied the DPM for a road with a known curvature. The vehicle kinematics for front-steering can be expressed by [18]:

$$
\frac{d}{d t}\left(\begin{array}{c}
x(t) \\
y(t) \\
v(t) \\
a(t) \\
\theta
\end{array}\right)=\left(\begin{array}{c}
v(t) \cdot \cos (\theta) \\
v(t) \cdot \sin (\theta) \\
a(t) \\
z(t) \\
\dot{\theta}(t)
\end{array}\right)
$$

where $x(t)$ and $y(t)$ are changes of the vehicle position. $v(t)$ is the value of speed, corresponding to the acceleration $a(t)$. Equivalently, $z(t)$ denotes the jerk which is derivative of accelerations. $\theta(t)$ refers the vehicle heading angle.

The simultaneous dynamic optimization was extensively used to solve constrained optimization problems subject to imposed constraints on the referred variables. Herein, the optimization of the speed planning can be expressed as a general nonlinear programming problem with equality and inequality constraints in accordance to [18],

$$
\min \sum h_{i}
$$

subject to,

1) the model principles for the vehicle speed and accelerations for $\forall t \in\left[0, t_{\mathrm{f}}\right]$

$$
\begin{aligned}
& v_{d}=v_{d-1}+a_{x_{i}} h_{i} \\
& a_{x_{i}}=a_{x_{i-1}}+z_{x_{i}} h_{i} \\
& a_{y_{i}}=a_{y_{i-1}}+z_{y_{i}} h_{i}
\end{aligned}
$$

2) the speed-curvature constraints $[17,19]$ for $\forall t \in$ $\left[0, t_{\mathrm{f}}\right]$

$$
v_{d} \leq \sqrt{\mathrm{p}_{1} /\left(k_{i}+\mathrm{p}_{2}\right)}
$$

3) constraints for driver's preference metric in $\forall t \in$ $\left[0, t_{\mathrm{f}}\right]$

$$
\begin{gathered}
a_{x_{i}} \leq a_{+x_{D P M}} \\
a_{x_{i}} \geq a_{-x_{D P M}} \\
a_{y_{i}} \leq\left|a_{y_{D P M}}\right| \\
z_{x_{i}} \leq|0.6| \\
z_{y_{i}} \leq|0.6|
\end{gathered}
$$

4) the linear relationship of longitudinal-lateral acceleration for $\forall t \in\left[0, t_{\mathrm{f}}\right]$

$$
\begin{gathered}
a_{x_{i}} \leq-\left(\frac{a_{x_{D P M}}}{a_{y_{D P M}}}\right) \cdot a_{y_{i}}+a_{x_{D P M}}\left(a_{x_{i}} \geq 0\right) a_{x_{i}} \\
a_{x_{i}} \geq\left(\frac{\left|a_{-x_{D P M}}\right|}{a_{y_{D P M}}}\right) \cdot a_{y_{i}}+a_{-x_{D P M}}\left(a_{x_{i}}<0\right)
\end{gathered}
$$

where $t_{\mathrm{f}}$ indicates a terminal moment for the entire optimization process. The symbol $v_{i}$ denotes the vehicle speed during $h_{i}$, which is the duration of the time element between $t_{i}$ and $t_{i-1}$, and $a_{x_{i}}$ and $a_{y_{i}}$ refer to the corresponding longitudinal and lateral accelerations. Equivalently, $z_{x_{i}}$ and $z_{y_{i}}$ are the longitudinal and lateral jerk corresponding to the accelerations.

The speed-curvature constraints is the safety conditions that the acquired speed must be satisfied. These are expressed as a function of the trajectory curvature $k_{i}$. Correspondingly, $\mathrm{p}_{1}$ and $\mathrm{p}_{2}$ are the parameters for Reymond model [17, 19], which constitutes an additional constraint used to prevent violations of the speed $\left(v_{c c}\right)$ on the curved road. These parameters were obtained from the fitted curve based on test data from human drivers.

The proposed discrete time optimization problem was solved with the A mathematical programming language (AMPL) using interior point optimizer (IPOPT) solver [20-22]. The minimum time speed profile $v_{d}$ can be obtained based on the Eq. (2)-(7) of the formulated dynamic optimization problem.

Fig. 3 describes an example of the optimization 

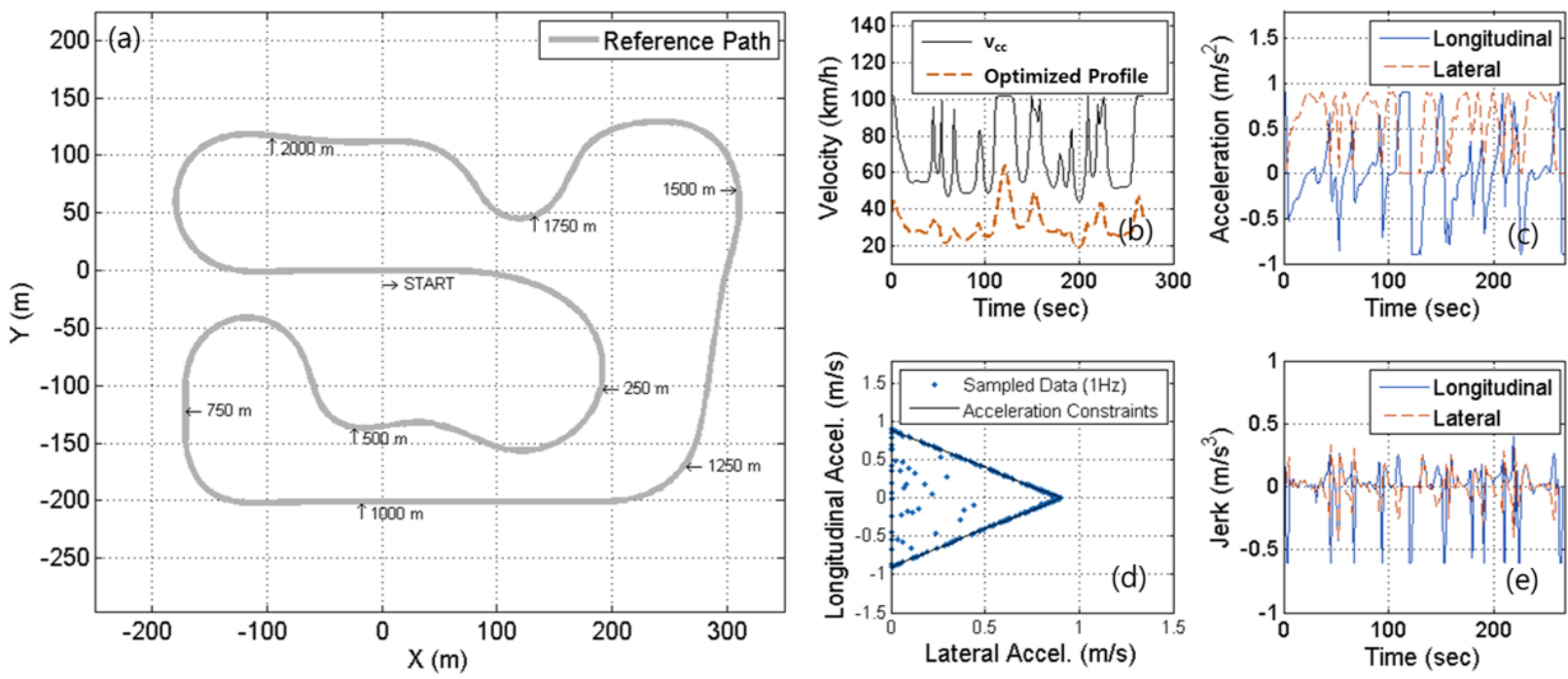

Figure. 3 Optimization results of the desired speed planning calculated for DPM $=\{0.9,-0.9,|0.9|,|0.6|,|0.6|\}:$ (a) reference path, (b) optimized speed profile $\left(v_{d}\right)$ and safety speed corresponding curvature constraint $\left(v_{c c}\right)$, (c) calculated lateral and longitudinal accelerations, and (d) calculated lateral and longitudinal jerks on a GG diagram, and (e) calculated lateral and longitudinal jerks

results of the speed planning based on the cautious driver preference. These simulated optimization results were assigned to region of $\mathrm{DPM}=\{0.9,-0.9$, $|0.9|,|0.6|,|0.6|\}$, and the initial and terminal speed conditions for $\forall t \in\left[0, t_{\mathrm{f}}\right]$ in accordance to

$$
\begin{aligned}
& v(0)=10(\mathrm{~m} / \mathrm{s}), a(0)=0 \\
& v\left(t_{\mathrm{f}}\right)=10(\mathrm{~m} / \mathrm{s}), a\left(t_{\mathrm{f}}\right)=0
\end{aligned}
$$

Fig. 3 (a) shows the test course with many curved sections, which was selected to demonstrate the optimization. The minimum time velocity profile and Reymond velocity model $\left(v_{c c}\right)$ with the recommended parameter $\left(\mathrm{p}_{1}=4.58 \mathrm{~m} / \mathrm{s}^{2}, \mathrm{p}_{2}=\right.$ $5.69 \times 10^{-3} \mathrm{~m}^{-1}$ ) are presented in Fig. 3 (b). In the simulation, the maximum velocity of the straight course was limited by $102 \mathrm{~km} / \mathrm{h}$, approximately. Fig. 3 (c), and (e) describe the calculated longitudinal and lateral motion profiles with the jerk and acceleration constraints. Fig. 3 (d) represents the calculated lateral and longitudinal jerks on the GG diagram.

As shown in Fig. 3, the optimal temporal speed profile was successfully obtained by satisfying the DPM constraints for a cautious driving style. The entire optimization process was conducted with an Intel core i7-2710Q processor with 8 GB memory. In this case, the expended computational time was approximately $0.09 \mathrm{~s}$ for the entire optimization process.

\subsection{Combined lateral and longitudinal controller}

The combined longitudinal and the lateral controller is required to track the planned trajectory with the desired speed for various driving states of the autonomous vehicle. Fig. 4 shows the simplified system block diagram of the combined longitudinal and lateral controller based on prior studies [23-26]. The proposed combined vehicular control system is based on the PID control and generates input signals such as steering, accel and brake pedal position in order to perform simultaneous path and speed tracking, while ensuring the dynamic stability and tracking performance of an autonomous vehicle. More details on the integrated longitudinal and lateral control system, can be invoked from [23-26].

\section{Experiments and results}

\subsection{On-vehicle hardware system}

For the experimental test of the proposed control system on a real road, we modified the commercial

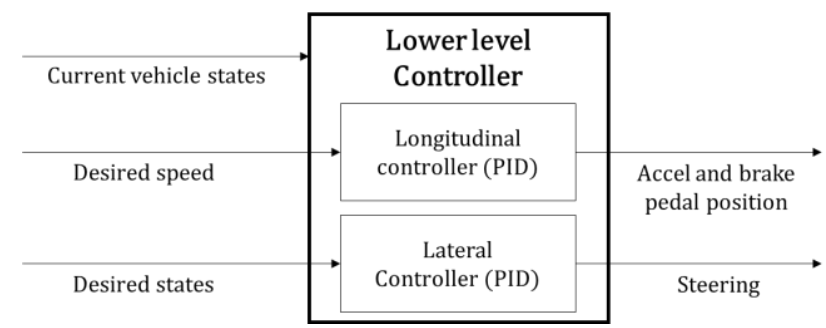

Figure. 4 Simplified system block diagram of the combined longitudinal and lateral controller 


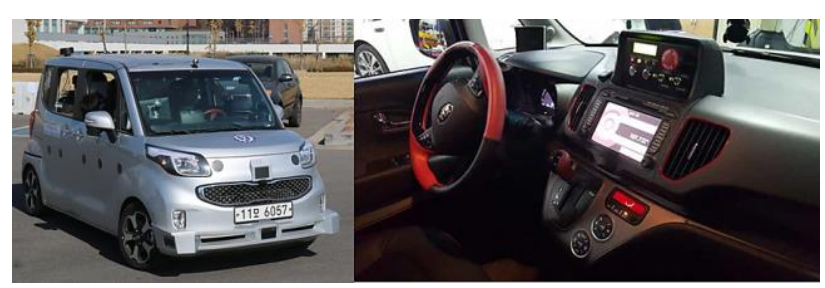

Figure. 5 Photograph of the autonomous vehicle used for the conducted road experiments

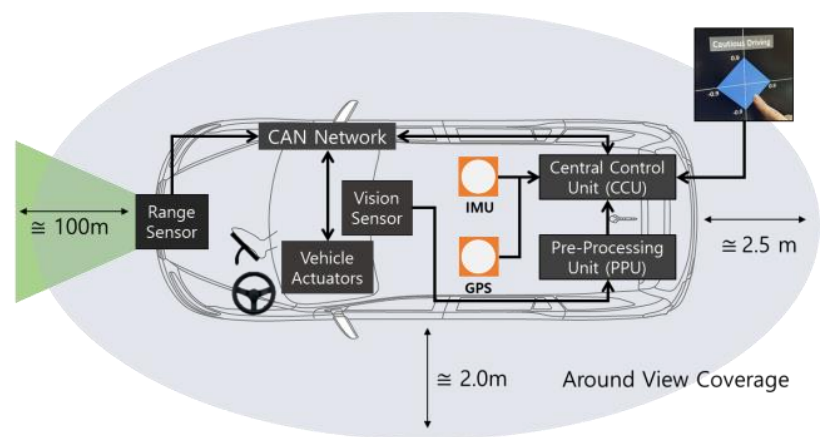

Figure. 6 Architecture of an autonomous vehicle equipped with the proposed control system

vehicle RAY from Kia motor company to an autonomous vehicle that was equipped with the multiple cameras, range sensors, actuators, and the proposed control scheme, as shown in Fig. 5 and Fig. 6. In spite of the compactness of the car, the modified test vehicle provided enough space for all on-board equipment. This vehicle has a gasolinepowered engine, front-wheel drive, and motordriven power steering (MDPS).

We developed the distributed computing system architecture that consists of the central processing unit (CCU) and pre-processing unit (PPU), as shown in Fig 6. A fan-less embedded computer (UMS5301 model) with an Intel Core i7-2710Q central processing unit was used as the central controller, and had enough computing power to perform path planning, and execute the overall control algorithm described in the previous sections. The proposed driver's preference setting interface has been implemented by NI LabVIEW with MATLAB script engine interacting with an in-vehicle touch monitor for the fast prototyping.

PPU consists of an Intel Core i7 $2.1 \mathrm{GHz}$ central processing unit, and it was mainly used for image processing to extract the meaningful environmental information for the vehicle using the stereo vision, and the around view monitoring camera system.

The vehicle's instantaneous state of speed, PRND gear position, angular speed of engine rotation, and steering wheel angle, were gathered from the in-vehicle CAN bus, and were then transferred to the CCU. The control commands of the steering angle and throttle maneuver of the vehicle were input to the vehicle through the CAN gateway network to control the vehicular actuators, such as the MDPS, a brake and an accel pedal position value. There was a separated actuator interface, but it was controlled by the $\mathrm{CCU}$ for maneuvering the gear shift lever and pressing the brake pedal.

The GPS antenna was mounted on the roof of the vehicle at the center of the rear axle, and it was also equipped with an IMU sensor (IG-500A model) to measure the dynamic motion of the vehicle, including the yaw angle, the lateral acceleration and longitudinal acceleration. We used the Novatel FlexFak6 GPS sensors which can support the Network-RTK to achieve high performance in terms of position accuracy, so that the position error tolerance was within $2.0 \mathrm{~cm}$. All data from vehiclemounted sensors were synchronized at $10 \mathrm{~Hz}$.

\subsection{Results of on-road experimental test}

We conducted an experimental test at the testing route, as shown by the satellite image map (Fig. 7 (a)). This testing course was approximately $1.3 \mathrm{~km}$ long, and included four intersections without interruptions from traffic lights. The route was suitable for the evaluation of the performance of the proposed DPM-based control system. In the realroad test, we set the DPM criterion to match the various driver's driving styles, while the specific DPM criterion selected for the test drive was appropriate for the normal driving style (labeled using the symbol \#FT), expressed as,

$$
D P M \# F T=\{0.6,-0.6,|1.5|,|0.6|,|0.6|\}
$$

Fig. 7 and Table 1 show the measured results of the field test for autonomous driving experiments. The acceleration data were acquired by the IMU sensor, and velocity and steering angle data were obtained through a CAN interface during the test.

Measured experimental data illustrate that vehicular velocity followed the planed velocity during driving. The measured acceleration and velocity profiles of the self-driving vehicle indicate a driving style that is similar to the heuristic pattern of deceleration-steering-acceleration. Fig. 7 (b) shows the recorded longitudinal and lateral accelerating profiles, where the vehicle decelerates while it approaches an intersection, and makes a turn before it accelerates again.

The measured lateral acceleration profile shows four overshoots coincident with the events 

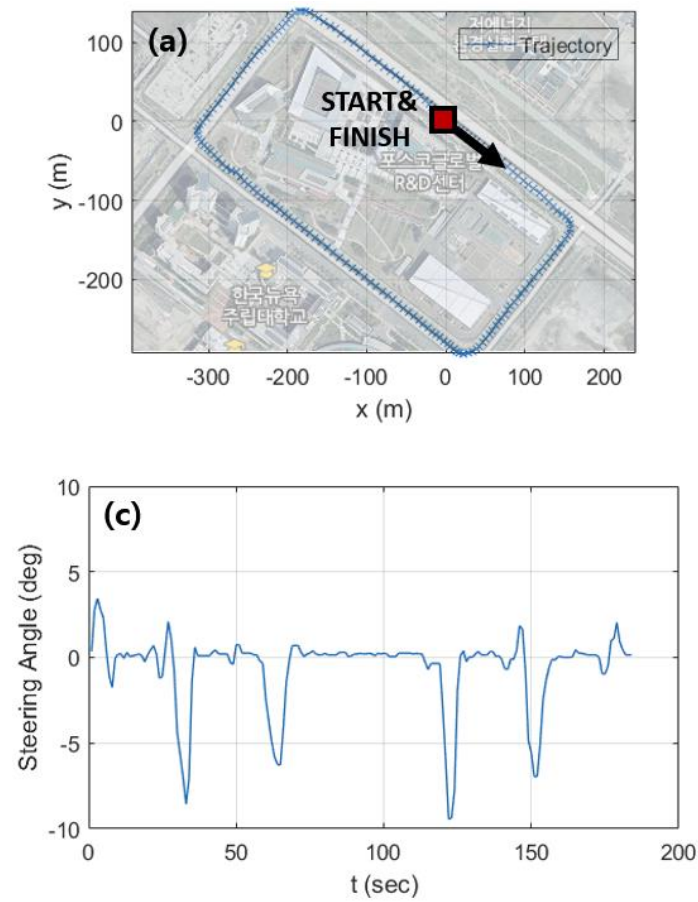
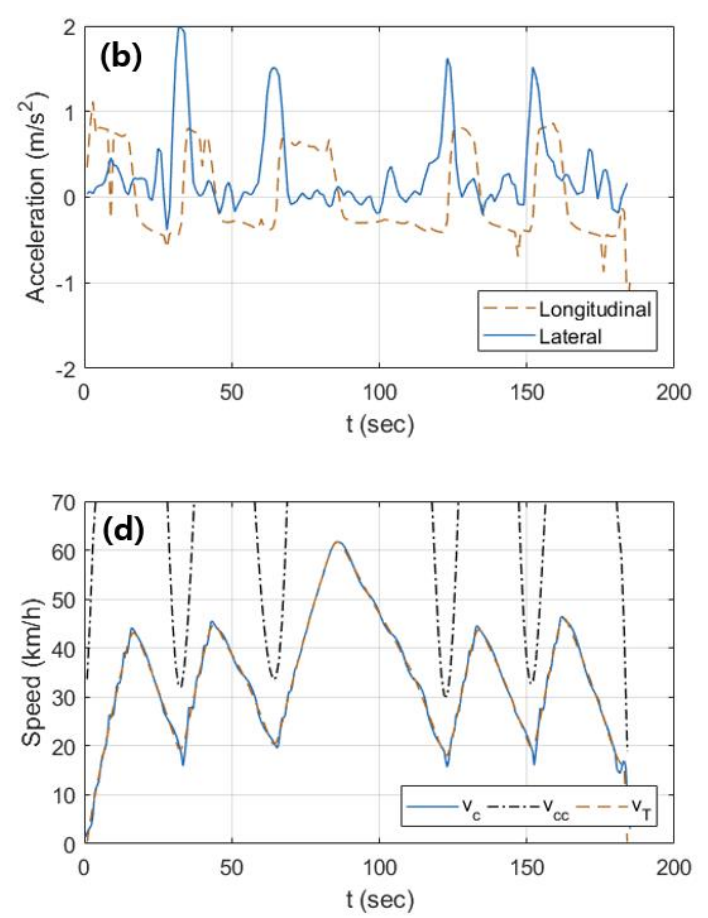

Figure. 7 Experimental results of the autonomous vehicle in accordance to the DPM criterion for a typical normal driver $(\mathrm{DPM} \# \mathrm{FT}=\{0.6,-0.6,|1.5|,|0.6|,|0.6|\})$ : (a) Satellite image map of the test route, (b) measured accelerations of the vehicle using the in-vehicle IMU (IG-500A) sensor, (c) measured front-steering angle, and (d) plots of time optimum velocity profile $\left(v_{T}\right)$, threshold speed of Reymond's model $\left(v_{c c}\right)$ and experimental data of vehicular speed $\left(v_{c}\right)$ as a function of time obtained from in-vehicle CAN during real-road tests

Table 1. Measured peak lateral accelerations in turning section during the real experiment

\begin{tabular}{|c|c|c|}
\cline { 2 - 3 } \multicolumn{1}{c|}{} & $\begin{array}{c}\text { Measured peak } \\
\text { acceleration }\left(\mathrm{m} / \mathrm{s}^{2}\right)\end{array}$ & $\begin{array}{c}\text { Deviation from } \\
\text { given DPM }\left(\mathrm{m} / \mathrm{s}^{2}\right)\end{array}$ \\
\hline $1^{\text {st }}$ turning & 1.93 & -0.43 \\
\hline $2^{\text {nd }}$ turning & 1.52 & -0.02 \\
\hline $3^{\text {rd }}$ turning & 1.55 & -0.05 \\
\hline $4^{\text {th }}$ turning & 1.48 & 0.02 \\
\hline
\end{tabular}

associated with changing steering angles for right turns at intersections. Table 1 shows measured peak lateral acceleration values and deviations from the DPM criterion. The peak value of deviation from the DPM criterion was $-0.43 \mathrm{~m} / \mathrm{s}^{2}$ at the first turn, but after the first turn, the proposed system controlled the vehicle less than the $3.3 \%$ deviation from the specified criteria of normal driving style in the experiment.

The measured value of the lateral acceleration seemed to be limited, but the values exceeded the given DPM criterion during turning maneuvers. Additionally, there were glitches in the measured longitudinal acceleration, but the overall values were regulated within the given criterion. This measured trend of the longitudinal and lateral acceleration values at the curved section of the route was consistent with the simulated results since only the lateral acceleration results slightly exceeded the criterion limit, while the longitudinal acceleration was within DPM boundary. Owing to a latency in the vehicle's velocity response with respect to the desired input velocity, the vehicular speed during turning was slightly higher than the planned velocity required to meet the criterion of lateral acceleration. This might be the main reason for which the lateral acceleration value was marginally outside the border line.

\section{Discussions}

The DPM criterion included constant lateral and longitudinal jerk value. However, we could not measure jerk in the experiments using the IMU sensor because of the measurement noise in the acceleration data acquisition. Jerk is the derivative of acceleration, and even low-noise amplitudes in the acceleration data can cause spikes in the estimation of jerk owing to the temporal derivative of the data. Although various studies have been conducted for the improvement of the online jerk sensor [27], or the estimation method [28], to the best of our knowledge, on-vehicle lateral or longitudinal jerk sensors for robust data acquisitions have not been commercialized thus far. Therefore, in the proposed controller, the jerk term can be taken into account only at the stage of the planning of the desired velocity. 
The limitation in the implementation of the proposed work is the in-situ jerk measurement onvehicle, and the consideration of other factors that influence ride quality of drivers other than acceleration and jerk (i.e., distance to the car ahead, and complexity of surroundings). Since the implementation of the complete DPM-aware controller requires an advancement in-vehicle sensors, detection and perception technologies, so this topic remains a theme for further research.

\section{Conclusions}

This study has been described a method to meet the driver's driving preference for a personalized and a comfortable automated driving experience for each user. For this purpose, we had investigated about the individual driving style and defined a driver's driving preference metric (DPM), which is specified preferred lateral and longitudinal acceleration region.

We also proposed a vehicular controller with combined lateral and longitudinal controller that utilized the DPM control parameters through the interacting with GG diagram interface for the customization, and aimed to provide DPM-aware control in autonomous vehicles. The overall structure of the proposed occupant's metric control system is simple and easy concept to implement in autonomous vehicles. The performance of the proposed control system was verified with the onroad experimental tests. The experimental result shows that the proposed DPM-aware system can control the lateral and longitudinal acceleration of autonomous vehicle within $28.7 \%$ of peak error tolerance from the specified criterion of DPM.

As a further research, we will improve the overall system performance to enable a robust speed control and to ensure the safety even in high speed conditions.

\section{Acknowledgments}

This research was supported by the MSIT (Ministry of Science and ICT), Korea, under the "ICT Consilience Creative Program" (IITP-2019-2017-001015) supervised by the IITP (Institute for Information \& Communications Technology Planning \& Evaluation). The authors performed this work as a part of research projects of SKT-Yonsei Cooperative Autonomous Driving Research Center supported by the SK Telecom ICT R\&D Center.

\section{References}

[1] M. Elbanhawi, M. Simic, and R. Jazar, "In the passenger seat: investigating ride comfort measures in autonomous cars", IEEE Intelligent Transportation Systems Magazine, Vol.7, No.3, pp.4-17, 2015.

[2] M. Sivak and S. Brandon, "Motion sickness in self-driving vehicles", The University of Michigan Transportation Research Institute, pp.1-13, 2015.

[3] S. Thrun, M. Montemerlo, H. Dahlkamp, D. Stavens, A. Aron, J. Diebel, P. Fong, J. Gale,M. Halpenny, G. Hoffmann, K. Lau, C. Oakley, M. Palatucci, V. Pratt, P. Stang, S. Strohband, C. Dupont, L. E. Jendrossek, C. Koelen, C. Markey, C. Rummel, J. van Niekerk, E. Jensen, P. Alessandrini, G. Bradski, B. Davies, S. Ettinger, A. Kaehler, A. Nefian, and P. Mahoney, "Stanley: The robot that won the DARPA Grand Challenge", Journal Field Robotics, Vol.23, No.9, pp.661-692, 2006.

[4] C. Urmson, J. Anhalt, J. A. D. Bagnell, C. R. Baker, R. E. Bittner, J. M. Dolan, D. Duggins, D. Ferguson, T. Galatali, H. Geyer, M. Gittleman, S. Harbaugh, M. Hebert, T. Howard, A. Kelly, D. Kohanbash, M. Likhachev, N. Miller, K. Peterson, R. Rajkumar, P. Rybski, B. Salesky, S. Scherer, Y.-W. Seo, R. Simmons, S. Singh, J. M. Snider, A. T. Stentz, W. R. L. Whittaker, and J. Ziglar, "Tartan racing: A multi-modal approach to the DARPA urban challenge", Robotics Institute, Carnegie Mellon University, 2007.

[5] M. Bojarski, D. Del Testa, D. Dworakowski, B. Firner, B. Flepp, P. Goyal, and X. Zhang, "End to end learning for self-driving cars", arXiv preprint arXiv:1604.07316, 2016.

[6] N. A. Spielberg, M. Brown, N. R. Kapania, J. C. Kegelman, and J. C. Gerdes, "Neural network vehicle models for high-performance automated driving", Sci. Robot. Vol.4, No.28, 2019.

[7] F. Hartwich, M. Beggiato, and J. F. Krems, "Driving comfort, enjoyment and acceptance of automated driving-effects of drivers' age and driving style familiarity", Ergonomics. Vol.61, No.8, pp.1017-1032, 2018.

[8] M. Kyriakidis, J. C. de Winter, N. Stanton, T. Bellet, B. van Arem, K. Brookhuis, M. H. Martens, K. Bengler, J. Andersson, N. Merat, and N. Reed, "A human factors perspective on automated driving", Theoretical Issues in Ergonomics Science. Vol.20 No.3, pp.223-49, 2019. 
[9] R. Vaiana, T. Iuele, V. Astarita, M. V. Caruso, A. Tassitani, C. Zaffino, and V. P. Giofrè, "Driving behavior and traffic safety: an acceleration-based safety evaluation procedure for smartphones", Modern Applied Science, Vol.8, No.1, 2014.

[10] F. Biral, M. Da Lio, and E. Bertolazzi, "Combining safety margins and user preferences into a driving criterion for optimal control-based computation of reference maneuvers for an ADAS of the next generation", In: Proc. of IEEE Intelligent Vehicles Symposium, 2005.

[11] L. Eboli, G. Mazzulla, and G. Pungillo, "Combining speed and acceleration to define car users' safe or unsafe driving behaviour", Transportation Research Part C: Emerging Technologies, Vol.68, pp.113-125, 2016.

[12] Y. Koh, H. Her, K. Yi, and K. Kim, "Integrated speed and steering control driver model for vehicle driver closed-loop simulation", IEEE Transactions on Vehicular Technology, Vol.65, No.6, pp.4401-4411, 2016.

[13] J. C. McCall and M. M. Trivedi, "Driver behavior and situation aware brake assistance for intelligent vehicles", In: Proc. of the IEEE, Vol.95, No.2, pp.374-387, 2007.

[14] C. Martinez, M. Heucke, F. WangY, B. Gao, and D. Cao, "Driving style recognition for intelligent vehicle control and advanced driver assistance: A survey", IEEE Transactions on Intelligent Transportation Systems, Vol.19, No.3, pp.666-676, 2017.

[15] W. Wang, J. Xi, and H, Chen, "Modeling and recognizing driver behavior based on driving data: A survey", Mathematical Problems in Engineering, 2014.

[16] M. John, "The GG diagram", 1996, Available from: http://hpwizard.com/gg-diagram.html

[17] P. Bosetti, L. Mauro, and S. Andrea, "On the human control of vehicles: an experimental study of acceleration", European Transport Research Review, Vol.6, No.2, pp.157-170, 2014.

[18] I. Bae, J. Moon, and J. Seo, "Toward a Comfortable Driving Experience for a SelfDriving Shuttle Bus", Electronics, Vol.8 No.9, 2019.

[19] G. Reymond, A. Kemeny, J. Droulez, and A. Berthoz, "Role of lateral acceleration in curve driving: Driver model and experiments on a real vehicle and a driving simulator", J. Hum. Factors Ergonom. Soc., Vol.43, No.3, pp.483495, 2001.
[20] A. Wachter and L. T. Biegler, "On the implementation of a primal-dual interior point filter line search algorithm for large-scale nonlinear programming", Mathematical Programming, Vol.106, No.1, pp.25-57, 2006.

[21] R. Lougee-Heimer, "The common optimization interface for operations research: promoting open-source software in the operations research community", IBM Journal of Research and Development, Vol.47, No.1, pp.57-66, 2003.

[22] MATLAB quick start - AMPL API, Available online:

https://ampl.com/api/1.2.2/matlab/quickstart.html

[23] I. Bae, J. Moon, J. Cha, and S. Kim, "Integrated lateral and longitudinal control system for autonomous vehicles", In: Proc. of IEEE International Conference on Intelligent Transportation Systems (ITSC), pp. 406-411, 2014.

[24] I. Bae, J. H. Kim, and S. Kim, "Steering rate controller based on curvature of trajectory for autonomous driving vehicles", In: Proc. of IEEE Intelligent Vehicle Symposium (IV), pp.1381-1386, 2013.

[25] R. Attia, O. Rodolfo, and B. Michel, "Combined longitudinal and lateral control for automated vehicle guidance", Vehicle System Dynamics, Vol.52, No.2, pp.261-279, 2014.

[26] L. Xu, Y. Wang, H. Sun, J. Xin, and N. Zheng, "Integrated longitudinal and lateral control for Kuafu-II autonomous vehicle", IEEE Transaction on Intelligent Transportation System, No.17, Vol.7, pp.2032-2041, 2015.

[27] M. Yamakado, N. Keiichiro, and T. Junya, "A yaw-moment control method based on a vehicle's lateral jerk information", Vehicle System Dynamics, Vol.52, No.10, pp.12331253, 2014.

[28] A. Bisoffi F. Biral, F. M. Da Lio, and L. Zaccarian, "Longitudinal jerk estimation for identification of driver intention", In: Proc. of the IEEE International Conference on Intelligent Transportation Systems (ITSC), 2015. 